This is an author produced version of a paper published in Current Topics in Microbiology and Immunolgy. This paper has been peer-reviewed but does not include the final publisher proof-corrections or journal pagination.

Citation for the published paper:

Johannesson M, Hultqvist M, Holmdahl R..

"Genetics of autoimmune diseases: a multistep process.

Current Topics in Microbiology and Immunolgy, 2006, Issue: 305, pp. 259-76.

Access to the published version may require journal subscription.

Published with permission from: Springer 


\title{
Genetics of Autoimmune Diseases: A Multistep Process
}

\author{
M. Johannesson, M. Hultqvist, R. Holmdahl \\ M. Johannesson, M. Hultqvist, R. Holmdahl (e-mail: rikard.holmdahl@med.lu.se) \\ Medical Inflammation Research, Sölvegatan 19, BMC I11, Lund University, S-22184 \\ Lund, Sweden
}

\begin{abstract}
It has so far been difficult to identify genes behind polygenic autoimmune diseases such as rheumatoid arthritis (RA), multiple sclerosis (MS), and type I diabetes (T1D). With proper animal models, some of the complexity behind these diseases can be reduced. The use of linkage analysis and positional cloning of genes in animal models for RA resulted in the identification of one of the genes regulating severity of arthritis in rats and mice, the Ncf1 gene. The Ncf1 gene encodes for the Ncf1 protein that is involved in production of free oxygen radicals through the NADPH oxidase complex, which opens up a new pathway for therapeutic treatment of inflammatory diseases. In most cases, however, a QTL is the sum effect of several genes within and outside the QTL, which make positional cloning difficult. Here we will discuss the possibilities and difficulties of gene identification in animal models of autoimmune disorders.
\end{abstract}

\section{Introduction}

The major autoimmune diseases such as rheumatoid arthritis (RA), multiple sclerosis, (MS) and type I diabetes (T1D) as well as related inflammatory disorders such as asthma, psoriasis, and atherosclerosis are complex, and it has been difficult to understand their fundamental genetic and environmental causes. With proper animal models genetic and environmental factors can be better controlled and also manipulated. This enables genetic approaches that lead to an insight not possible to achieve with direct human 
studies only. The possibility of combining the genetic knowledge with direct animal studies will enhance the understanding of these diseases and shorten the time needed to develop new therapies with higher efficiency and limited side-effects. We will discuss these possibilities, mainly using examples from recent studies of models for RA.

\section{Definition of Autoimmune Disease: Physiology Versus Pathology}

Identification of genes controlling autoimmune diseases requires that we have a clear definition of what an autoimmune disease is. Over the years, this definition has been shifting and also varies depending on whether it is seen from a clinical point of view or from a basic science point of view.

From a clinical point of view, these diseases are inflammatory diseases of unknown origin, which cannot be explained by any obvious cause such as infection or allergen exposure. To strengthen the classification they should be chronic, i.e., they should last for a period that exceeds a normal acute inflammatory phase of usually 3- 4 weeks. The occurrence of autoantibodies targeting some relevant tissue antigen is often, but not always, included as an additional criteria . However, the pathogenic relevance of such antibodies is not always clear. These definitions have led us to classify a number of commonly occurring chronic diseases as classical autoimmune, for example MS, RA, T1D, and systemic lupus erythematosus (SLE). A borderline group is made up of inflammatory diseases with a possible but not proven infectious cause such as spondyloarthropathies and inflammatory bowel diseases. Another borderline group comprises diseases in which an inflammatory component has emerged as one of the major driving factors, for example, psoriasis and atherosclerosis. It is obvious that from this perspective autoimmune diseases compose a highly heterogeneous group, even within each disease classification, as there are not yet means to link diagnosis with disease mechanisms. Rather we are bound to description of the more obvious pathologic 
end-result, such as the lack of insulin in T1D or the neurological deficit in MS.

From the basic science standpoint, autoimmune recognition is often the phenomenon of interest for definition of autoimmune diseases. It stems from a tradition of viewing autoimmunity as forbidden self or "horror autotoxicus," as Burnett described it. The view has been broadened over the years with inclusion of innate immune recognition, or more colorfully expressed as "danger," as a factor not only in combat of infections but also in playing a role in the development of autoimmunity. The immune recognition is seen as the essential key for understanding both regulatory/suppressor and helper/effector mechanisms. Autoimmunity is defined through autoimmune recognition by lymphocytes, an event that is physiologic but results in an autoimmune disease when such lymphocytes are pathogenic. Proof for autoimmunity can be tested in animal models but not in humans, which has led to the development of animal models for autoimmune diseases. The models are mainly of two types: one that is induced and one where the disease develops spontaneously. Of the induced models, many are induced through immunization with an autoantigen, and the pathogenicity of autoreactive lymphocytes can be clearly shown. In general, only the acute phase of the first inflammatory response is studied. Examples of induced models are collagen-induced arthritis (CIA) as a model for RA [1, 2] and experimental autoimmune encephalomyelitis (EAE) as a model for MS [3]. Other models develop "spontaneously" due to genetic aberrations. In the spontaneous models, as in humans, the pathogenicity of specific autoimmune recognition is often difficult to document and the disease course is often chronic. But in contrast to humans, these models are often monogenic, highly penetrant and less influenced by environmental factors. Examples of spontaneous models are the transgenic models for arthritis (TNFa [4], IL1Ra deficiency [5], anti-glucose-6-phosphoisomeras T cell receptor [6]), various spontaneous lupus models (NZB xNZW, MRL//pr, BXSB) [7] and diabetes in the NOD mouse [8].

Clearly, discrepancies between results from studies of autoimmune diseases between 
human and mouse are not only influenced by the species differences but are in most cases due to different disease definitions and methods. A better definition of the human diseases and also more appropriate, well-characterized animal models are therefore needed.

\section{From Genes to Disease: The Basic Science Approach}

The weakness of the basic science approach is the difficulties to clearly define the pathology of the phenomenon. In search of the pathologic effect of the mechanisms, numerous experiments has been carried out in animal models for human diseases where basically any disturbance of the physiology after knocking out a hypothesized gene is described as pathological. Clearly, in a complex system such as the immune system, the result will be pathologic in most cases, but the question remains, what is relevant for a naturally selected disease as occurring in humans? And how do we know that the postulated genes, proteins, mechanisms, or pathways are indeed relevant and essential for an autoimmune disease in humans? One way to find out is to first identify the genes involved in the disease development. Then, when we have defined the most critical genes and thereby the most critical pathways, the basic approaches to studying the molecular interacting pathways will be crucial for understanding the disease.

\section{From Disease to Genes: The Monogenic Success and the Polygenic Failure}

The cause of autoimmune diseases seems to be easily delivered by genetics. Why not just define the genes carried by diseased individuals and then determine their functional genetics? Is this not just a question of adding resources into the human genetic project and its prolongations in the form of various strategic research platforms? The human genome project has indeed been fruitful and we have had enormous success with hundreds of monogenic diseases. One example in the autoimmune field is the 
identification of the AIRE (autoimmune regulator) gene causing the APECED syndrome $[9,10]$. The APECED syndrome is a familiar disease characterized by a combination of fungal infections and autoimmune inflammation of various endocrine organs. It is a monogenic disease with high penetrance and caused by mutations in the AIRE gene. This discovery did not only identify the gene behind APECED but also led to a renewed understanding of autoimmunity as the $A / R E$ gene plays an important role in the induction of central tolerance through its expression in the thymic epithelial cells [11]. However, polymorphism in AIRE could not explain the major autoimmune diseases that are more complex and polygenic in nature. Genetic linkage analysis of large familiar cohorts has been relatively unsuccessful. A few, relatively highly penetrant genes, such as NOD2 [12] and PTPN22 [13], have been replicated in several association studies, but these are likely to reflect only the tip of the iceberg. What we need is a more complete picture of the genetic architecture, with all the genes involved, high- as well as low-penetrant, and to know how they interact with each other and with the environment. However, even when the association with a gene or haplotype is known, it can still be difficult to find the actual function. One example of the difficulties is the MHC complex. Most autoimmune diseases have been known for decades to be associated with various strongly conserved $\mathrm{MHC}$ haplotypes. However, it has not been possible in any disease to clearly identify the gene(s) within the haplotypes, although there is circumstantial evidence for the importance of certain class II genes (DR4/DR1 with the shared epitope) in RA [14] and class I genes (B27) in spondyloarthropathies [15].

It is likely that more of the genetic secrets in humans will be revealed by adding resources, as done through the HapMap project and other efforts. However, the approach requires substantial resources and the question is whether we can make shortcuts and what complementary approaches we can use. The use of improved animal models for human diseases would be a major advantage in several aspects. The environmental factors could be controlled and we have inbred strains simplifying the genetics and the 
linkage analysis, strains that can be genetically manipulated and thereby allow us to test our hypotheses (Fig. 1).

\section{The Use of Animal Models in a Disease-to-Gene Approach}

Experience from studies on animal models of autoimmune diseases show that they are much more efficient and appropriate for linkage analysis than human cohorts. The process of positional cloning involves five generic steps that intend to lead from phenotype to gene discovery (described in Fig. 2). In general, an F2 intercross of 100400 animals will reveal loci explaining $10 \%-50 \%$ of a given disease trait and also allows for studying some of the genetic interactions involved. Thus, the power is much higher compared to human studies. What we have learned from animal models for autoimmune diseases so far is that there are a multitude of genes of variable penetrance controlling a disease, and in addition, there are complex genetic interactions and also interactions with the environment. It is important to remember that, depending on the phenotype, the number of loci, their relative effects, and how they interact with each other and the environment varies considerably. Most likely, the complexity revealed in the genetic studies of animal models will also apply for humans. Therefore, the definition of both genetic and environmental interactions are critical for finding the underlying genes causing and controlling autoimmune diseases, a task that is also difficult in animal models, here illustrated by two examples: the positional cloning of the Pia4 gene Ncf1 and the complex structure and interactions of the Eae3 and Eae2 loci.

Position Cloning of Ncf1, a Genetic Polymorphism Explaining a Major Quantitative Trait Locus Controlling Chronic Inflammation Pristane-induced arthritis (PIA) in rats is a model mimicking RA. Arthritis is induced by a subcutaneous injection of $100-200 \mu$ of the adjuvant oil pristane at the base of the tail and signs of arthritis appear after about 14 days. The subsequent development of arthritis is chronic and relapsing in the susceptible DA strain, with an erosive and symmetric 
destruction of peripheral joints and the disease fulfils the classical RA criteria [16, 17]. PIA is $\mathrm{T}$ cell-dependent and there is no evidence for an influence of B cells or arthritogenic antibodies in contrast to CIA, the primarily used arthritis model in mice, in which arthritogenic antibodies to CII play a significant role [18].

To identify the genes contributing to the disease, the arthritis-susceptible DA rat was crossed with the arthritis-resistant E3 rat $[19,20]$. In a number of both intercrosses and backcrosses involving more than 1,000 rats, the major quantitative trait loci (QTLs) could be identified (Fig. 3). These loci could clearly be separated to control specific phases of the disease course, i.e., onset, severity, and chronicity (Fig. 4). One of the QTLs, Pia4, located on chromosome 12, was found to be associated with severity of PIA. By insertion of the genetic fragment of interest from the resistant strain (in this case E3) into the genome of the susceptible DA strain through conventional backcross breeding (more than ten generations), we created a congenic strain. At this stage, the congenic fragment was $20 \mathrm{cM}$ and we found that the congenic rat developed much milder arthritis than the parental DA rat. In fact, the difference was significant using fewer than ten rats in each group, which is a criterion for going further to clone the underlying gene/s. In other words, the prerequisite was that we had transformed the trait to be more Mendelian. On this basis, we started a process of collecting and testing small cohorts of animals having recombinations within this fragment in order to establish subcongenic strains [21]. Each new subcongenic strain was screened for the presence of the disease-associated genes by testing them for PIA susceptibility. With this method, the fragment could be reduced to contain only two genes, Ncf1 and Gtf2i. No differential expression of either Ncf1 or Gtf2i could be detected and only Ncf1 (alias p47phox) had polymorphism alterations in the coding sequence leading to changes in amino acid sequence in the translated protein, suggesting that Ncf1 was essential for arthritis susceptibility. One of the alterations was at position 106 ATG/GTG and resulted in a Met/Val alteration, the other one was at position 153 ATG/ACG and resulted in a Met/Thr alteration. Brown Norway (BN) rats, which are 
resistant to arthritis, share all genotypes but one with DA, the alteration in position 153 , suggesting that this alteration is important for the Ncf1 function. Consequently, this approach allowed us to identify a causative gene, which could now be subjected for further functional analysis and studies of this new pathogenic pathway associated with arthritis severity.

\section{The Ncf1 Protein and the NADPH Complex}

How could the Ncf1 gene be involved in the development of arthritis? To our advantage, a huge effort in investigation and characterization had already been made regarding the Ncf1 gene and the encoded Ncf1 protein. The fact that a lot of information was already available simplified the process of finding phenotypes associated with the genotype. The Ncf1 protein is part of the NADPH oxidase complex expressed in phagocytic cells. The complex is composed of five subunits, of which two, Cyba (alias gp91phox) and Cybb (alias alias p22 phox), resides in the membrane where they form a heterodimeric flavohemoprotein—cytochrome $b_{558}$. The other three subunits—Ncf1, Ncf2 (alias p67phox) and Ncf4 (alias p40phox)—are in the resting state forming a complex in the cytosol, but when activated by any of a wide variety of stimuli, the Ncf1 protein gets heavily phosphorylated and the complex migrates to the membrane to form the activated complex [22]. The Ncf1 protein is the subunit responsible for transporting the cytosolic complex to the endosomal membrane or the cell membrane during activation [23]. The NADPH oxidase complex functions as an electron donor in the oxidative burst process activated in response to pathogenic invaders, ultimately generating reactive oxygen species (ROS) [24]. To investigate the functional consequences of the identified Ncf1 polymorphism, we tested the ability of rat peritoneal cells to produce ROS. The surprising results showed that the arthritis-susceptible DA rat had a lower burst than the resistant DA. $N c f^{E 3}$ congenic rat [21]. One allele containing the functional $N c f 1$ was enough to correct for the deficiency in oxidative burst and protect from disease since the congenic 
heterozygous for the $\mathrm{Ncf}^{E 3}$ allele had a milder disease than the homozygous DA rat. These results contradict the general belief that oxidative burst promotes inflammation and suggest that the oxidative burst is involved in a previously unknown pathway controlling inflammation. In fact, transfer of spleen-derived T cells from the congenic DA. $N c f^{E 3}$ rat to the parental DA and vice versa showed that the Ncf1 polymorphism operated before activation of the $T$ cells, indicating that it plays a role in the control of autoimmune activation.

\section{Pathway Analysis}

Natural selection does not operate on single genes but rather on molecular pathways. Thus, the discovery of a genetic polymorphism, like in Ncf1, regulating a disease trait, opens up the possibility for the discovery of a new pathway leading to arthritis. The pathways are likely to be shared between different species; however, the exact nucleotide variant in the rat Ncf1 gene is probably specific. Thus, for further studies it can be assumed that the pathway will operate similarly in rats, mice, and humans.

To investigate this possibility we looked to the mouse. In the mouse there is an arthritis QTL identified that contains the Ncf1 gene. However, we found that Ncf1 was not polymorphic and we did not find an effect on the oxidative burst using a congenic strain with a fragment derived from $\mathrm{C} 3 \mathrm{H}$ on a B10.Q background (P. Olofsson et al., unpublished data). Another mouse that was made deficient for Ncf1 was not possible to use for our purpose, as the mutation results in a complete destruction of the gene. Moreover, the Ncf1-targeted mutation also contains a genetically linked 129-derived fragment [25], known to contain other polymorphic genes of importance for arthritis [26]. However, a worldwide search through available mouse strains identified a variant of a C57B1/6 strain with mutations in both the leptin receptor and the Ncf1 gene [27]. The point mutation in the Ncf1 gene is located at position - 2 in exon 8 and results in an aberrant splicing of the Ncf1 mRNA transcripts. One of the transcripts resulting from this mutation give rise to 
low levels of a protein that is truncated, lacking eight amino acids in the second $\mathrm{SH} 3$ domain that are important for interaction with the Ncf2 protein, thus resulting in absence of detectable NADPH oxidase activity. Consequently, we now had another mutation that presumably should affect the same pathway as was discovered in the rat. The C57BI/6 mouse with the mutation in the Ncf1 gene was therefore backcrossed to the arthritissusceptible B10.Q strain. The mice were tested for susceptibility to CIA, where arthritis is induced by an intradermal injection of rat collagen type II (CII) emulsified in complete Freund's adjuvant. The B10.Q mice homozygous for the mutation in Ncf1 developed severe arthritis with chronic development, i.e., similar to the low oxidative burst responder DA rat strain [28]. Mice heterozygous for the mutation were found to develop a less severe arthritis than Ncf1-mutated mice resembling the situation in rats where one functional Ncf1 allele is enough to correct both oxidative burst and disease severity. Interestingly, both the delayed type hypersensitivity (DTH) and antibody response to CII were higher in the Ncf1 mutated mice, indicating an increased activity of Cll reactive $\mathrm{T}$ cells. We also found that some female Ncf1 mutated mice in the breeding colony spontaneously developed arthritis. In an experiment, 3 out of 11 female mice developed severe arthritis a few days after partus, confirming earlier findings that there is a high risk for arthritis susceptibility during the postpartum period [29]. The arthritis was severe and chronic, as in CIA, and the mice with spontaneous arthritis also had a higher level of antibodies against CII with the same fine-specificity as in CIA [28].

The overall data clearly suggest another role of NADPH oxidase-derived ROS than just elimination of pathogens, adding a complexity to the redox status of the cell. ROS response has been reported not only in phagocytes but also in antigen-presenting cells (APCs), such as dendritic cells (DCs) [30]. Also, T cells have been reported to express Ncf1 during antigen presentation and activation [31], adding further complexity to the process of finding the mechanisms behind the phenotype.

The immune regulatory effect of ROS could be involved in one or more of the different 
stages of the interactions of the immune system. There are a number of pathways that are proposed to be affected by free radicals and state of oxidation. On the antigenpresenting level, it has been shown that by inhibiting ROS production, the proliferation of T cells in response to antigen presentation of DCs is prevented [30], suggesting that the burst response in an APC could modulate the antigen presentation capacity of that cell. The level of radicals could also directly act on T cells. ROS could possibly be transferred over the synapse from APC to T cell and thereby alter the oxidation state of the recipient cell. It has been suggested that the level of oxidation on membranes of cells, influenced by interacting cells, are important for function [32]. Extracellular ROS has also been shown to act as an immune regulator through its interaction with the $\mathrm{T}$ cell proliferation regulator NO [33]. The oxidation could also possibly act by affecting other important structures on the cell surface and thereby alter their status. It has been shown that oxidation on T cell receptor signaling pathways, such as the linker for activation of T cells (LAT) molecule, modulates the function of T cells [34]. It is also possible that ROS by itself acts as a modulator of the system. It is known that ROS in low concentrations serves as a second messenger in the initiation and amplification of signaling at the antigen receptor in lymphocyte activation (reviewed in [32]). Another possibility is that ROS is involved in feedback mechanisms. Direct cell- cell contact between an activated T cell and human PMN has been shown to induce intracellular ROS production in the PMN, which then could act as an intracellular messenger [35]. Further studies are needed in order to clarify which effect is of importance for the development of arthritis.

\section{Therapeutic Possibilities Can Be Immediately Explored}

Taken together these data show that it is possible to identify the underlying genes of a

QTL. It also shows that the importance of this finding is the identification of new pathogenic pathways and that these pathways are conserved between species. The NADPH oxidase complex proved to be an important regulator of the immune system and 
the pathway appears to involve autoreactive T cells, but it is not yet fully understood. This new pathway for modulating inflammatory diseases is, however, expected to open up new possibilities for generating drugs to target chronic inflammatory autoimmune diseases such as arthritis. Most importantly, using the animal model platform including the congenic strains, methods are then already prepared for testing proof of concepts of the different therapeutic possibilities. This will contribute to shorten the time from the target identification to validation.

\section{Analyses of a QTL Containing Interacting Genes}

Several factors affected the successes of the positional cloning of Ncf1. First of all, it is a gene with a large effect size, accounting for about $20 \%$ of the total phenotypic variation [19]. Moreover, the mutation has a dominant effect on a well-characterized phenotype and also, the rest of the genome in the DA background allows the functional expression of the allele. However, in many cases a QTL effect is the sum effect of several positionally linked genes and their interactions with other genes both within and outside the QTL. This certainly makes positional cloning more difficult.

One such example is two of the first QTLs reported to control an animal model of an autoimmune disease: the Eae2 and Eae3 loci associated with experimental autoimmune encephalomyelitis [36]. Eae2 and Eae3 are located on chromosomes 3 and 15, respectively, and these loci have an interacting effect on the disease [36]. We made congenic strains with these fragments from the resistant RIIIS/J strain on the susceptible B10.RIII background. The effect on disease in these mice was mild, far from being Mendelian and therefore not suitable for positional cloning of the underlying genes. Clearly both the environmental and genetic conditions needed to be better defined in order to increase the effect of the RIIIS/J alleles [37, 38]. Firstly we found that both loci also controlled collagen-induced arthritis. Moreover, studying environmental factors affecting the arthritis phenotype we found that inducing the disease without tubercle 
antigens in the adjuvant increased the penetrance of the congenic effect in both chromosome 15 and chromosome 3 congenics. We also utilized the observation that these loci interacted and developed a strategy to define the genetic interactions. The congenics were intercrossed for more than eight generations, resulting in a partial advanced intercross cohort of roughly 1,000 mice. This accumulated both the recombination density and statistical power to dissect the loci into several subloci and to identify the interactions between them. Within the previous QTL on chromosome 3 (Eae3), we were able to identify three separate loci (Cia5, Cia21, and Cia22) and within the previous QTL on chromosome 15 (Eae2) we found four loci (Cia26, Cia30, Cia31, and Cia32). Most of these loci interacted with each other (Fig. 5) and RIIIS/J alleles at the different loci could be either disease-promoting or disease-decreasing. For example; Cia5 affected the onset and early severity of arthritis in additive interaction with Cia30 on chromosome 15, whereas the Cia21 and Cia22 affected severity during the chronic phase of the disease in epistatic interaction with Cia31 on chromosome 15. The definition of the environmental conditions and the genetic interactions was a prerequisite to dissect the Eae2 and Eae3 QTLs. Human studies will have the same problem, but they are more readily seen and analyzed in animal model studies. In the case of the Eae2/Eae3 project, the interactions between the QTLs could be detected in the F2 intercross with no more than approximately 100 animals. However, the breakdown of the two QTLs into seven and detecting the specific interactions among them was only possible by collecting a larger number of recombinations in approximately 1,000 mice. What we learned was that by analyzing the genetic interactions in the $\mathrm{F} 2$ intercross we could get information on how to design congenic strains with the optimal effect on the phenotype. The knowledge of the genetic interactions is then not only useful in the positional cloning process but also once the gene is identified and studied in humans.

The interactions within a QTL are another case and they pose both new problems and possibilities. The problems are the difficulties in splitting up a tightly linked haplotype. This 
is an apparent problem in human studies, for example in addressing conserved haplotypes within the MHC region. In animal models, haplotypes can be intentionally broken up through the search of recombinations. Such recombinations will often lead to new and changed effects on the phenotype, possibly caused by a balancing interaction between closely positioned genes. It will be a challenging task to investigate in detail such clusters but it is also important to remember that the minimal congenic fragment, containing such conserved haplotypes, are likely to have been selected for a balanced control of a specific pathway. Such congenic strains will therefore be of value for studies of pathophysiologic pathways and thereby for understanding the fundamental laws determining the possibility of developing drugs with efficiency and with limited sideeffects.

The possibility of genetically modifying mouse strains through transgene and embryonic stem cell-based technologies is a major advantage in the further dissection of the critical genes and haplotypes. Studies on the role of MHC genes are an excellent example of this. The establishment of $\mathrm{H}-2$ (i.e., mouse $\mathrm{MHC}$ ) congenic strains was the basis for the identification of the MHC regions and such strains are still useful for studies of the role of MHC. It has, however, been difficult to split this region in order to isolate each gene and thereby understand its function. Instead, transgenic experiments have been conducted with which it has been possible to insert both specific murine class II genes $[39,40]$ and their human counterparts [41-44], and thereby prove the role of these genes for the development of autoimmune disease such as RA and MS. These studies also show the risk in transgenic technology as overexpression of class II genes may also lead to an artifactual toxicity in B cells $[45,46]$, which is the most likely explanation for a suppressive effect by transgenic class II genes, as has been reported in diabetes in the NOD mouse.

\section{Conclusions}

It is essential to backtrack the work of nature, and disease-causing factors need to be 
searched for in the balance between a naturally but historically selected genome and newly introduced environmental factors. Due to the complexity of the major diseases, it has been difficult to follow an approach to make a direct search for the genetic and environmental factors causing autoimmune diseases. We have been arguing for the usefulness of proper animal models, with which it is possible to make a shortcut for the identifications of the major genetically selected pathways leading to autoimmune disease. Once identified the strength of the well-developed tools for experimental studies can be used. In combination this will shorten the time and efforts to develop new types of treatment addressing the disease-causing mechanisms.

\section{Acknowledgements}

Grant supporters were the Craaford foundation, the Lundberg foundation, the Kock and Österlund foundations, the Swedish Association Against Rheumatism, the Swedish Medical Research Council, the Swedish Foundation for Strategic Research, Arexis AB, and the European Union Grant EUROME QLG1-CT2001- 01407.

\section{References}

1. Trentham DE, Townes AS, Kang AH (1977) Autoimmunity to type II collagen an experimental model of arthritis. JExp Med 146:857

2. Courtenay JS, Dallman MJ, Dayan AD, Martin A, Mosedale B (1980) Immunisation against heterologous type II collagen induces arthritis in mice. Nature 283:666

3. Holmdahl R (1999) Genetic control of experimental models of multiple sclerosis. In: H Links (ed) Advances in multiple sclerosis. Clinical research and therapy. Martin Dunitz, London, p 15

4. Keffer J, Probert L, Cazlaris H, Georgopoulos S, Kaslaris E, Kioussis D, Kollias G (1991) Transgenic mice expressing human tumour necrosis factor: a predictive genetic 
model of arthritis. EMBO J 10:4025

5. Horai R, Saijo S, Tanioka D, Nakae S, Sudo K, Okahara A, Ikuse T, Asano M, Iwakura Y (2000) Development of chronic inflammatory arthropathy resembling rheumatoid arthritis in interleukin 1 receptor antagonist-deficient mice. J Exp Med 191:313

6. Kouskoff V, Korganow AS, Duchatelle V, Degott C, Benoist C, Mathis D (1996) Organspecific disease provoked by systemic autoimmunity. Cell $87: 811$

7. Theofilopoulos AN, Dixon FJ (1985) Murine models of systemic lupus erythematosus. Adv Immunol 37:269

8. Delovitch TL, Singh B (1997) The nonobese diabetic mouse as a model of autoimmune diabetes: immune dysregulation gets the NOD. Immunity 7:727

9. Nagamine K, Peterson P, Scott HS, Kudoh J, Minoshima S, Heino M, Krohn KJ, Lalioti MD, Mullis PE, Antonarakis SE, Kawasaki K,Asakawa S, Ito F, Shimizu N (1997)

Positional cloning of the APECED gene. Nat Genet 17:393

10. Aaltonen J, Björses P, Perheentupa J, Horelli-Kuitunen N, Palotie A, PeltonenL, Lee YS, FrancisF, Hennig S, Thiel C, Lehrach H, Yapso M-L (1997) An autoimmune disease APECED, caused by mutations in a novel gene featuring two PHD-type zinc-finger domains. Nat Genet 17:399

11. Liston A, Lesage S, Wilson J, Peltonen L, Goodnow CC (2003) Aire regulates negative selection of organ-specific T cells. Nat Immunol 4:350

12. Hugot JP, Chamaillard M, Zouali H, Lesage S, Cezard JP, Belaiche J, Almer S, Tysk C, O'Morain CA, Gassull M, Binder V, Finkel Y, Cortot A, Modigliani R, Laurent-Puig P, Gower-Rousseau C, Macry J, Colombel MSahbatou JF, Thomas G (2001) Association of NOD2 leucine-rich repeat variants with susceptibility to Crohn's disease. Nature 411:599 13. Begovich AB, Carlton VE, Honigberg LA, Schrodi SJ, Chokkalingam AP, Alexander HC, Ardlie QHuang KG, Smith AM, Spoerke JM, Conn MT, Chang M, Chang SY, Saiki RK, Catanese JJ, Leong DU, Garcia VE, McAllister LB, Jeffery DA, Lee AT, Batliwalla F, Remmers E, Criswell LA, Seldin MF, Kastner DL, Amos CI, Sninsky JJ, Gregersen PK 
(2004) A missense single-nucleotide polymorphism in a gene encoding a protein tyrosine phosphatase (PTPN22) is associated with rheumatoid arthritis. Am J Hum Genet 75:330 14. Gregersen PK, Silver J, Winchester RJ (1987) The shared epitope hypothesis. An approach to understanding the molecular genetics of susceptibility to rheumatoid arthritis. Arthritis Rheum 30:1205

15. Benjamin R, Parham P (1990) Guilt by association: HLA-B27 and ankylosing spondylitis. Immunol Today 11:137

16. Vingsbo C, Sahlstrand P, Brun JG, Jonsson R, Saxne T, Holmdahl R (1996) Pristaneinduced arthritis in rats: a new model for rheumatoid arthritis with a chronic disease course influenced by both major histocompatibility complex and non-major histocompatibility complex genes. Am J Pathol 149:1675

17. Arnett FC, Edworthy SM, Bloch DA et al (1988) The American Rheumatism Association 1987 revised criteria for the classification of rheumatoid arthritis. Arthritis Rheum 31:315

18. Holmdahl R, Rubin K, Klareskog L, Larsson E, Wigzell H (1986) Characterization of the antibody response in mice with type II collagen-induced arthritis, using monoclonal anti-type II collagen antibodies. Arthritis Rheum 29:400

19. Vingsbo-Lundberg C, Nordquist N, Olofsson P, Sundvall M, Saxne T, Pettersson U, Holmdahl R (1998) Genetic control of arthritis onset, severity and chronicity in a model for rheumatoid arthritis in rats. Nat Genet 20:401

20. Olofsson P, Holmberg J, Pettersson U, Holmdahl R (2003) Identification and isolation of dominant susceptibility loci for pristane-induced arthritis. J Immunol 171:407

21. Olofsson P, Holmberg J, Tordsson J, Lu S, Akerstrom B, Holmdahl R (2003) Positional identification of Ncf1 as a gene that regulates arthritis severity in rats. Nat Genet 33:25

22. Groemping Y, Lapouge K, Smerdon SJ, Rittinger K (2003) Molecular basis of phosphorylation-induced activation of the NADPH oxidase. Cell 113:343 
23. Heyworth PG, Curnutte JT, Nauseef WM, Volpp BD, Pearson DW, Rosen H, Clark RA (1991) Neutrophil nicotinamide adenine dinucleotide phosphate oxidase assembly. Translocation of p47-phox and p67-phox requires interaction between p47-phox and cytochrome b558. J Clin Invest 87:352

24. Babior BM (2000) Phagocytes and oxidative stress. Am J Med 109:33

25. Jackson SH, Gallin JI, Holland SM (1995) The p47phox mouse knock-out model of chronic granulomatous disease. J Exp Med 182:751

26. Blom T, Franzen A, Heinegard D, Holmdahl R (2003) Comment on "The influence of the proinflammatory cytokine, osteopontin, on autoimmune demyelinating disease".

Science 299:1845; author reply 1845

27. Huang CK, Zhan L, Hannigan MO, Ai Y, Leto TL (2000) P47(phox)-deficient NADPH oxidase defect in neutrophils of diabetic mouse strains C57BL/6J-m db/db and db/+. J Leukoc Biol 67:210

28. Hultqvist M, Olofsson P, Holmberg J, Backstrom BT, Tordsson J, Holmdahl R (2004) Enhanced autoimmunity, arthritis, and encephalomyelitis in mice with a reduced oxidative burst due to a mutation in the Ncf1 gene. Proc Natl Acad Sci U S A 101:12646

29. Mattsson R, Mattsson A, Holmdahl R, Whyte A, Rook GA (1991) Maintained pregnancy levels of oestrogen afford complete protection from post-partum exacerbation of collagen-induced arthritis. Clin Exp Immunol 85:41

30. Matsue H, Edelbaum D, Shalhevet D, Mizumoto N, Yang C, Mummert ME, Oeda J, Masayasu H, Takashima A (2003) Generation and function of reactive oxygen species in dendritic cells during antigen presentation. J Immunol 171:3010

31. Jackson SH, Devadas S, Kwon J, Pinto LA, Williams MS (2004) T cells express a phagocyte-type NADPH oxidase that is activated after T cell receptor stimulation. Nat Immunol 5:818

32. Reth M (2002) Hydrogen peroxide as second messenger in lymphocyte activation. Nat Immunol 3:1129 
33. Van der Veen RC, Dietlin TA, Karapetian A, Holland SM, Hofman FM (2004) Extracellular superoxide promotes $\mathrm{T}$ cell expansion through inactivation of nitric oxide. $\mathrm{J}$ Neuroimmunol 153:183

34. Gringhuis SI, Breedveld FC, Verweij CL (2002) Linker for activation of T cells: sensing redox imbalance. Methods Enzymol 352:248

35. Cettour-Rose P, Nguyen TX, Serrander L, Kaufmann MT, Dayer JM, Burger D, RouxLombard P (2005) T cell contact-mediated activation of respiratory burst in human polymorphonuclear leukocytes is inhibited by high-density lipoproteins and involves CD18. J Leukoc Biol 77:52

36. Sundvall M, Jirholt J, Yang HT, Jansson L, Engstrom A, Pettersson U, Holmdahl R (1995) Identification of murine loci associated with susceptibility to chronic experimental autoimmune encephalomyelitis. Nat Genet 10:313

37. Karlsson J, Johannesson M, Lindvall T, Wernhoff P, Holmdahl R, Andersson A (2005) Genetic interactions in Eae2 control collagen-induced arthritis and the CD4+/CD8+ T cell ratio. J Immunol 174:533

38. Johannesson M, Karlsson J, Wernhoff P, Nandakumar KS, Lindqvist AK, Olsson L, Cook AD, Andersson A, Holmdahl R (2005) Identification of epistasis through a partial advanced intercross reveals three arthritis loci within the Cia5 QTL in mice. Genes Immun $6: 175$

39. Brunsberg U, Gustafsson K, Jansson L, Michaelsson E, Ahrlund-Richter L, Pettersson RMattsson S, Holmdahl R (1994) Expression of a transgenic class IIAb gene confers susceptibility to collagen-induced arthritis. Eur J Immunol 24:1698 40. Kjellen P, Jansson L, Vestberg M, Andersson A, Mattsson R, Holmdahl R (2001) The $\mathrm{H} 2-\mathrm{Ab}$ gene influences the severity of experimental allergic encephalomyelitis induced by proteolipoprotein peptide 103- 116. J Neuroimmunol 120:25 41. Rosloniec EF, Brand DD, Myers LK, Whittington KB, Gumanovskaya M, Zaller DM, WoodsA, Altmann DM, Stuart JM, Kang AH (1997) An HLA-DR1 transgene confers 
susceptibility to collagen-induced arthritis elicited with human type II collagen. J Exp Med $185: 1113$

42. Rosloniec EF, Brand DD, Myers LK, Esaki Y, Whittington KB, Zaller DM, Woods A, Stuart JM, Kang AH (1998) Induction of autoimmune arthritis in HLA-DR4 (DRB1*0401) transgenic mice by immunization with human and bovine type II collagen. J Immunol 160:2573

43. Andersson EC, Svendsen P, Svejgaard A, Holmdahl R, Fugger L (2000) A molecule basis for the HLA association in rheumatoid arthritis. Rev Immunogenet 2:81

44. Madsen LS, Andersson EC, Jansson L, Krogsgaard M, Andersen CB, Engberg J, Strominger, Svejgaard A, Hjorth JP, Holmdahl R, Wucherpfennig KW, Fugger L (1999) A humanized model for multiple sclerosis using HLA-DR2 and a human T-cell receptor. Nat Genet 23:343

45. Singer SM, Umetsu DT, McDevitt HO (1996) High copy number I-Ab transgenes induce production of IgE through an interleukin 4-dependent mechanism. Proc Natl Acad Sci U S A 93:2947

46. Labrecque N, Madsen L, Fugger L, Benoist C, Mathis D (1999) Toxic MHC class II beta chains. Immunity 11:515

\section{Figure legends}

Fig. 1 The advantages of using animal models for genetic studies

Fig. 2 The five generic steps in positional cloning of quantitative trait genes. The first step is to map QTLs to chromosomal segments, usually in an F2 intercross or N2 backcross. This also involves the selection of inbred strains different for the phenotype studied. The number of F2 or N2 progeny genotyped and phenotyped determines the size of the linked regions and also sets the detection limits for how weak QTLs that can be found. The second step is to genetically isolate single QTLs and to measure the phenotype effect of each. This is usually done in a congenic strain. If the effect is reproduced in the congenic strain, then the third step is to reduce the size of the critical region as much as possible. 
Before molecular studies can be undertaken for gene identification, the linked region must be reduced to a few $\mathrm{Mb}$, preferably less. The fourth step is then to identify and evaluate candidate genes with different methods, all dependent on the identity of the genes. The fifth and final step is to establish proof of identity of the candidate genes with, for example, gene targeting, transgenic mice, or other functional tests

Fig. 3 Known regions in the rat genome linked to PIA

Fig. 4 Different loci control separate phases of PIA

Fig. 5 a A schematic view of the genetic interactions between the Eae2 and Eae3 loci affecting arthritis. b The sum of arthritis score in female mice depending on their genotypes at Cia21 and Cia32 


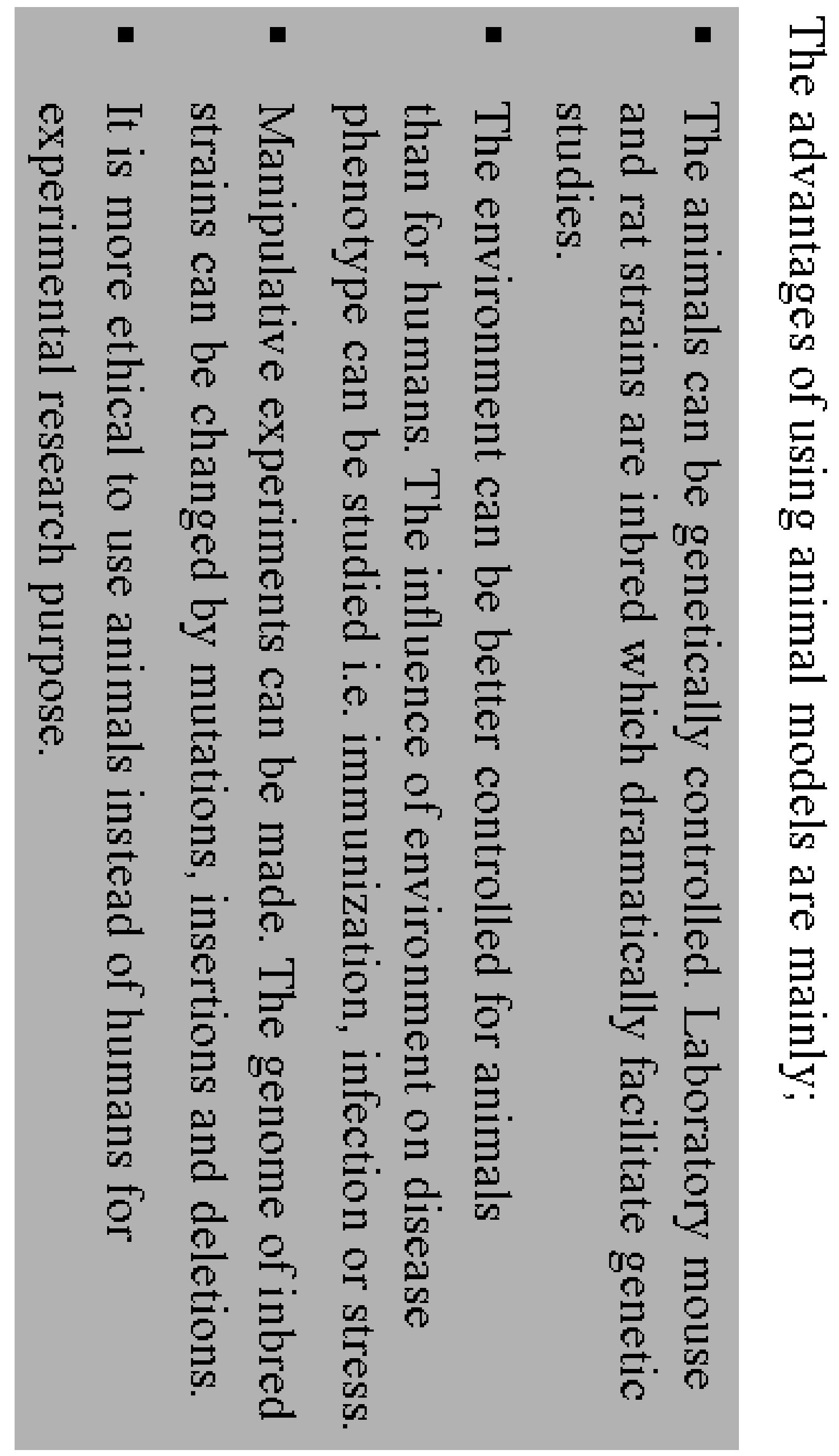




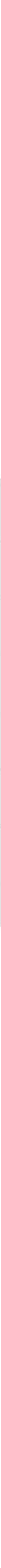




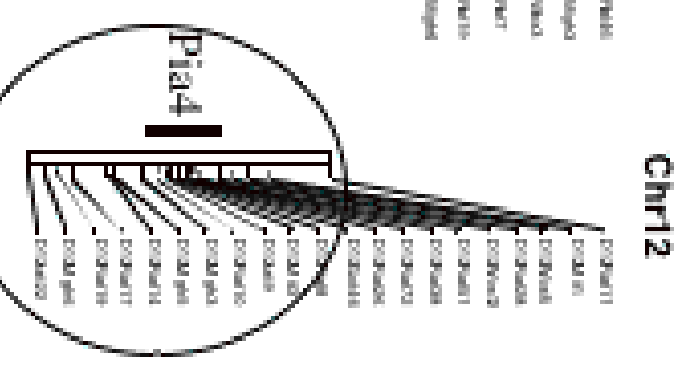

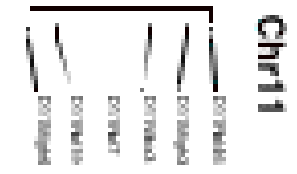
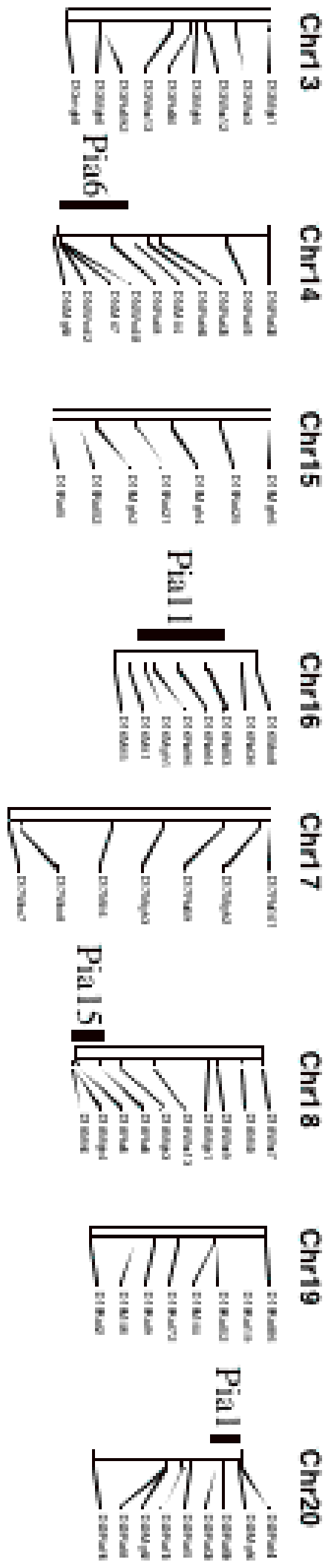

WMin EDE
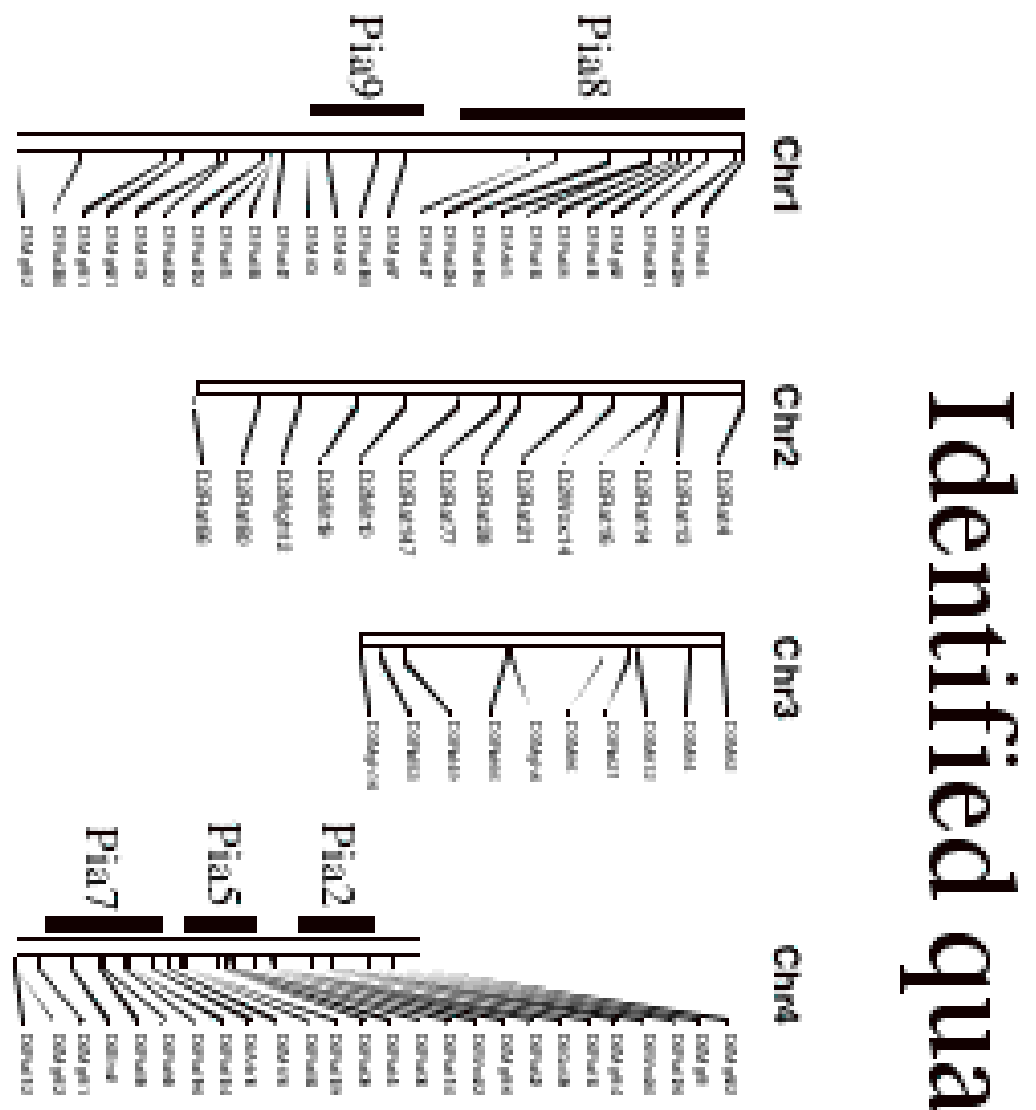

$\frac{9}{3}$

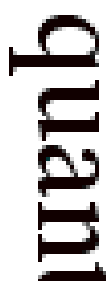

Thanh

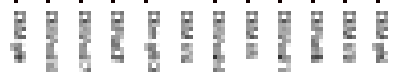
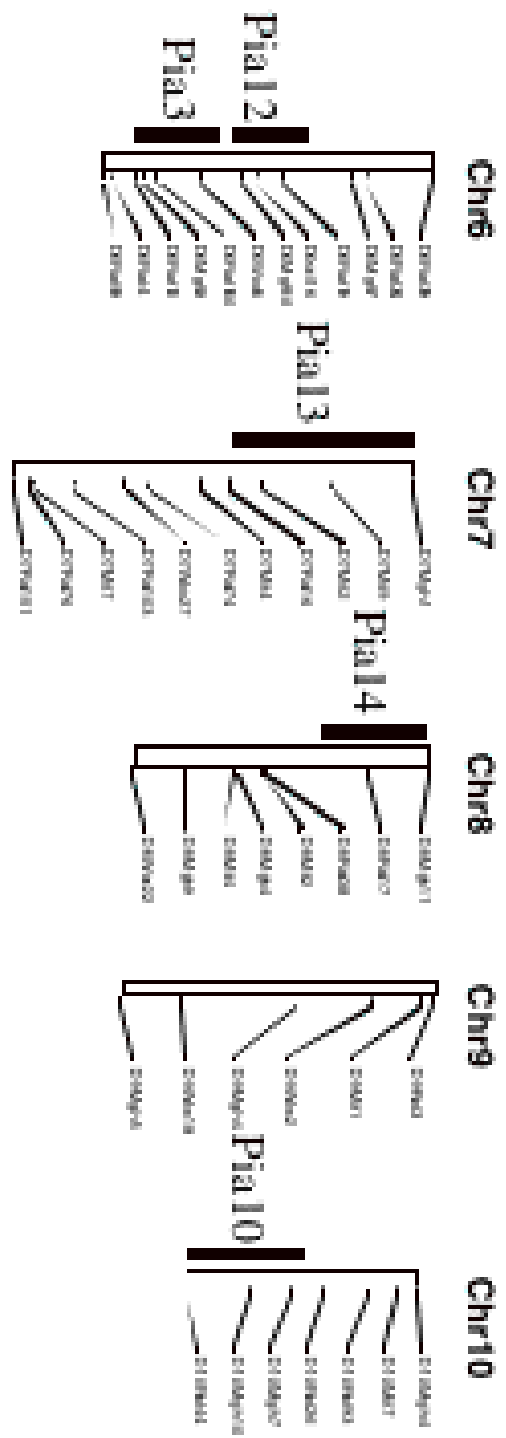
a)

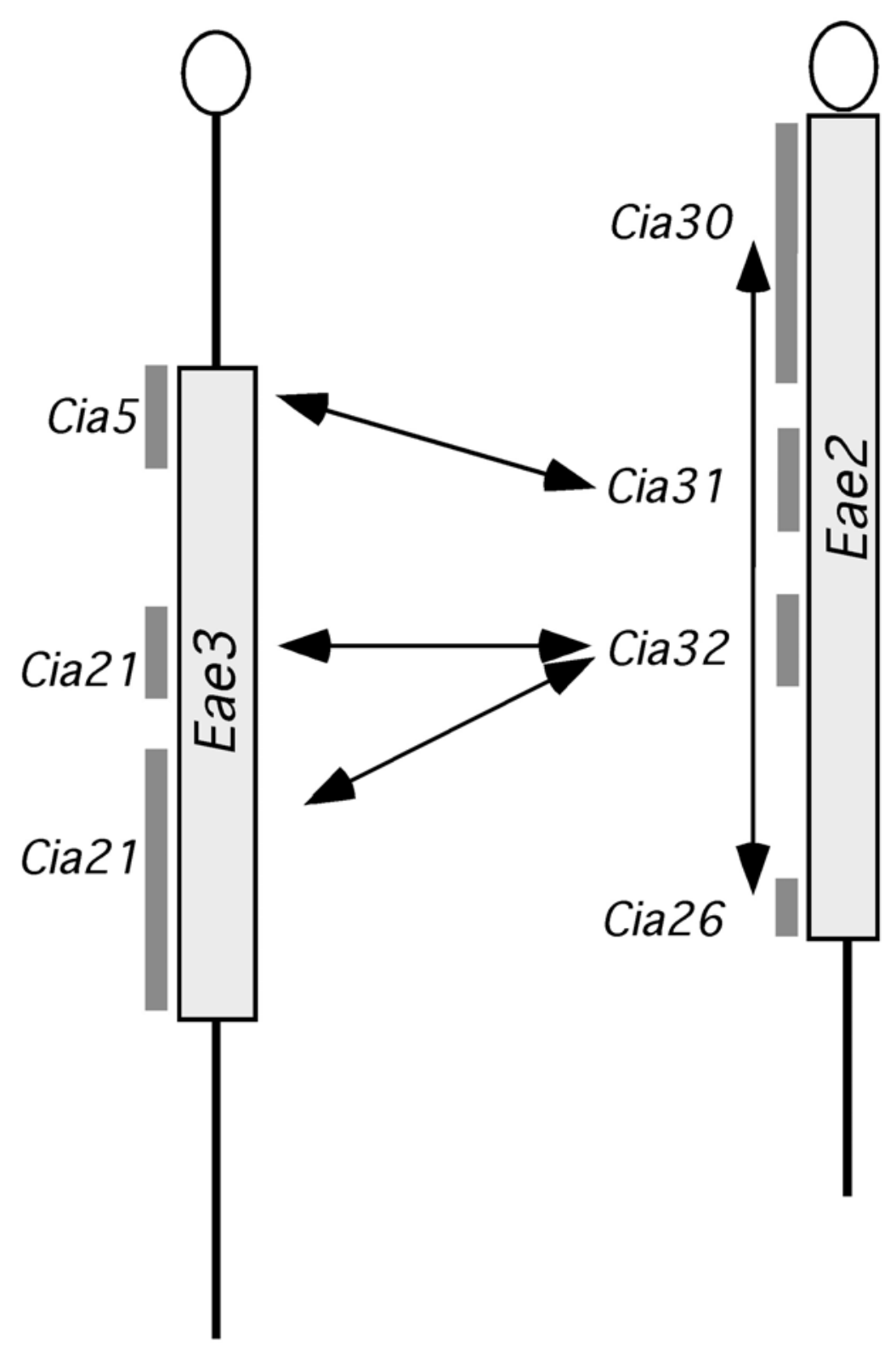

b)

Arhtritis severity

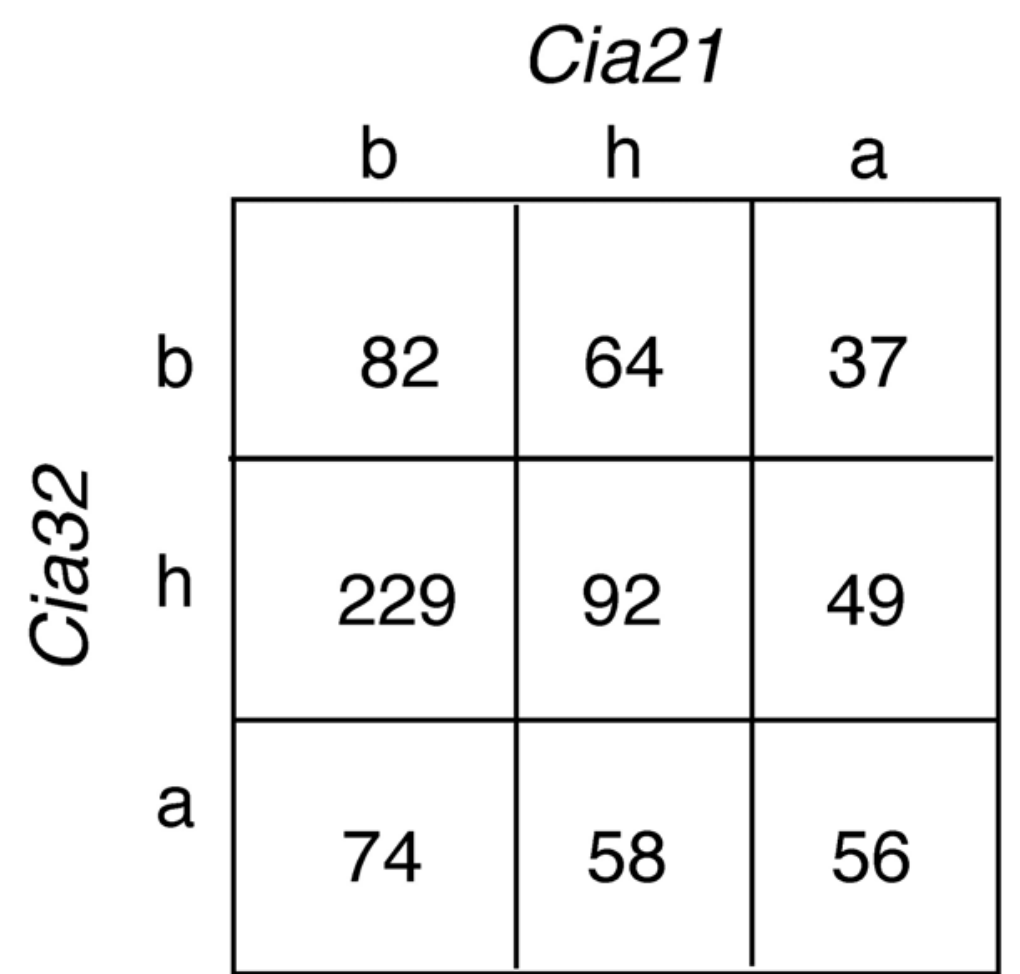

